\title{
3D Hierarchical Co-Al Layered Double Hydroxides with Long- Term Stabilities and High Rate Performances in Supercapacitors
}

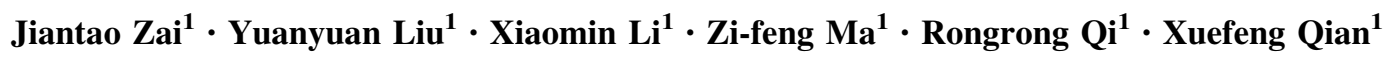

Received: 25 September 2016/Accepted: 10 November 2016/Published online: 27 December 2016

(C) The Author(s) 2016. This article is published with open access at Springerlink.com

\section{Highlights}

- 3D Flower-like Co-Al layered double hydroxides (Co-Al-LDHs) built up of atomically thin nanosheets were successfully synthesized via a hydrothermal method in a mixed solvent of water and butyl alcohol.

- Owing to the unique hierarchical structure and modification by butyl alcohol, the electrochemical stability and the charge/mass transport of the Co-Al-LDHs was improved, therefore leading to high specific capacitance, excellent rate performance and good cycling stability in supercapacitors.

\begin{abstract}
Three-dimensional (3D) flower-like Co-Al layered double hydroxide (Co-Al-LDH) architectures composed of atomically thin nanosheets were successfully synthesized via a hydrothermal method in a mixed solvent of water and butyl alcohol. Owing to the unique hierarchical structure and modification by butyl alcohol, the electrochemical stability and the charge/mass transport of the Co-Al-LDHs was improved. When used in supercapacitors, the obtained Co-Al-LDHs deliver a high specific capacitance of $838 \mathrm{~F} \mathrm{~g}^{-1}$ at a current density of $1 \mathrm{~A} \mathrm{~g}^{-1}$ and excellent rate performance $\left(753 \mathrm{~F} \mathrm{~g}^{-1}\right.$ at $30 \mathrm{~A} \mathrm{~g}^{-1}$ and $677 \mathrm{~F} \mathrm{~g}^{-1}$ at $100 \mathrm{~A} \mathrm{~g}^{-1}$ ), as well as excellent cycling stability with $95 \%$ retention of the initial capacitance even after 20,000 cycles at a current density of $5 \mathrm{~A} \mathrm{~g} \mathrm{~g}^{-1}$. This work provides a promising alternative strategy to enhance the electrochemical properties of supercapacitors.
\end{abstract}

Electronic supplementary material The online version of this article (doi:10.1007/s40820-016-0121-5) contains supplementary material, which is available to authorized users.

Rongrong Qi

rrqi@sjtu.edu.cn

Xuefeng Qian

xfqian@sjtu.edu.cn

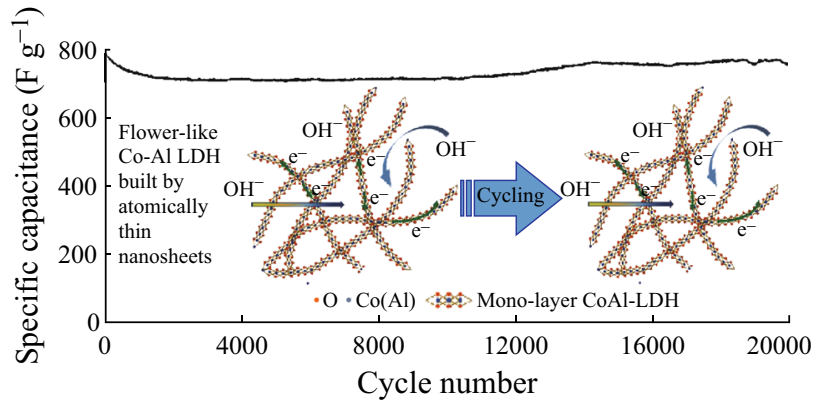

Keywords $\mathrm{Co}-\mathrm{Al}$ layered double hydroxides (Co-AlLDHs) - Nanosheets · 3D hierarchical architectures · Butyl alcohol $\cdot$ Supercapacitors

\section{Introduction}

To meet the increasing demand for clean energy technologies, many energy storage and conversion devices, such as fuel cells, batteries, and supercapacitors, have been developed [1-5]. Compared with other chemical energy

Shanghai Electrochemical Energy Devices Research Center, School of Chemistry and Chemical Engineering and State Key Laboratory of Metal Matrix Composites, Shanghai Jiao Tong University, Shanghai 200240, People's Republic of China 
storage devices, supercapacitors have attracted extensive attention owing to their fast charge/discharge rate, high power density, and long cycle lifetime [6-10]. Up to now, carbon-based capacitors have been widely studied due to their cost-effectiveness and excellent rate and cyclic stability [6]. However, the relatively low capacitance $\left(<300 \mathrm{~F} \mathrm{~g}^{-1}\right)$ cannot meet the demand for high energy density.

It has been reported that pseudocapacitive transition metal oxides/hydroxides possess high capacitances derived from their reversible faradic reactions [11-14]. Layered double hydroxides (LDHs), which are made up of positively charged brucite-like layers with an interlayer region containing charge compensating anions and solvation molecules, are promising electrode materials for supercapacitors due to the synergistic effects of bi-metal cations, such as reciprocal activation [15, 16]. However, the migration of metal cations can be limited by other cations, which can suppress the aggregation and growth of the active materials $[17,18]$. Co-Al-LDHs with divalent $\mathrm{Co}^{2+}$ ions and trivalent $\mathrm{Al}^{3+}$ ions are one of the most commonly studied LDHs because of their excellent electrochemical properties [19-21]. However, the specific capacitance, rate capability, and stability are usually poor because of the limited conductivity and the re-stacking of 2D nanosheets [22, 23]. Compositing with highly conductive substrates, such as $\mathrm{Ni}$ foil or carbon materials, is considered an effective method to improve the performance of Co-AlLDHs. For example, the porous Co-Al-LDHs/GO (GO, graphene oxide) nanocomposite exhibits a specific capacitance of $1043 \mathrm{~F} \mathrm{~g}^{-1}$ at $1 \mathrm{~A} \mathrm{~g}^{-1}$ [24]. H-OH intercalated Co-Al-LDHs on Ni foil shows a capacitance of $1031 \mathrm{~F} \mathrm{~g}^{-1}$ at $1 \mathrm{~A} \mathrm{~g}^{-1}$ and an ultrahigh rate capability with $66 \%$ capability retention at $100 \mathrm{~A} \mathrm{~g}^{-1}$ [25]. However, the cycling stability of LDHs is usually less than 5000 cycles (Table S1), which is far from the practical demand of 100,000-200,000 cycles. Therefore, the stability of Co-AlLDHs is the most prominent problem to overcome.

In general, active materials for electrodes with larger surface areas show higher capacitances and stabilities. Two-dimensional (2D) monolayer LDH nanosheets with extremely large surface areas can be prepared by a topdown method, in which LDH nanoplates are first prepared and then exfoliated in liquid medium by ultrasonic treatment [26]. However, the nanosheets prefer to re-stack to reduce the surface free energy, which is detrimental to the capacitance and stability of the electrodes. It has been accepted that three-dimensional (3D) hierarchical structures composed of 2D nanosheets are more stable than 2D nanosheets $[27,28]$. The unique structure is beneficial to charge and mass transport and the mitigation of volume change during the charge/discharge process [29]. Furthermore, 3D hierarchical structures can supply more points to connect the conductive matrix in the electrodes, which can provide more electron paths and suppress the separation of active materials [30-32]. On the other hand, the stability of the layered compounds can be improved by modification with organic compounds because they can intercalate and/ or adsorb into the layers to reduce the surface energy [33-36] and further prevent the re-stacking of nanosheets [37]. For example, Xiao et al. found that $\mathrm{MoS}_{2} / \mathrm{PEO}$ [poly(ethylene oxide)] nanocomposites had high reversible capacities with long-term reversibility because the incorporation of PEO can stabilize the disordered structure of $\mathrm{MoS}_{2}$ [38].

Herein, 3D hierarchical Co-Al-LDHs were fabricated in a rationally designed reaction system. Owing to the unique hierarchical structures composed of atomically thin nanosheets and the modification by butyl alcohol, the electrochemical stability and the charge/mass transport of the 3D Co-Al-LDH architectures were improved. When used in supercapacitors, high specific capacitance and good cycling stability were achieved.

\section{Experimental Section}

\subsection{Synthesis of 3D Hierarchical Co-Al-LDHs}

In a typical procedure, $\mathrm{Co}\left(\mathrm{NO}_{3}\right)_{2} \cdot 6 \mathrm{H}_{2} \mathrm{O} \quad(2.4 \mathrm{mmol}$, $0.698 \mathrm{~g})$ and $\mathrm{Al}\left(\mathrm{NO}_{3}\right)_{3} \cdot 9 \mathrm{H}_{2} \mathrm{O}(0.8 \mathrm{mmol}, 0.3 \mathrm{~g})$ were dissolved in $40 \mathrm{~mL}$ deionized water and $40 \mathrm{~mL}$ butyl alcohol and stirred for $30 \mathrm{~min}$. Then, $0.384 \mathrm{~g}$ of urea and $15 \mathrm{mg}$ of citric acid trisodium salt dehydrate were added and further stirred for another $30 \mathrm{~min}$. Next, the mixtures were sealed in a $100-\mathrm{mL}$ Teflon-lined steel autoclave and hydrothermally treated at $120{ }^{\circ} \mathrm{C}$ for $12 \mathrm{~h}$. After being cooled to room temperature naturally, the samples were filtered and washed with deionized water and ethanol several times and then freeze-dried $\left(5 \times 10^{-2} \mathrm{mbar}\right.$ at $\left.T \leq-46{ }^{\circ} \mathrm{C}\right)$ for $24 \mathrm{~h}$ to obtain the 3D Co-Al-LDHs. For comparison, 2D Co-AlLDHs were prepared using deionized water as the solvent, and zero-dimensional (0D) Co-Al-LDHs were prepared using butyl alcohol as the solvent under similar reaction conditions.

\subsection{Material Characterization}

The crystal structure and phase were characterized on an X-ray powder diffractometer (XRD, Shimadzu-6000) and X-ray photoelectron spectrometer (XPS, VG Scientific ESCLAB 220iXL). The size and morphology of the assynthesized products were determined by a transmission electron microscope (TEM, JEOL-1200) and field emission scanning electron microscope (FESEM, JEOL, JSM$7401 \mathrm{~F}$ ) with an accelerating voltage of $5 \mathrm{kV}$. Atomic force microscopy (AFM) measurements were collected on a 
Multimode atomic force microscope (Veeco Instruments, Inc.). Typically, a freshly diluted ethanol solution of the $\mathrm{NiFe}-\mathrm{LDH}$ samples was ultrasonically treated and then deposited onto a clean mica wafer by drop-casting. The nitrogen adsorption-desorption measurement was conducted on a Micromeritics ASAP 2010 analyzer, and the specific surface areas of samples were determined by Brunauer-Emmett-Teller (BET) analysis. FT-IR spectra were recorded on a PerkinElmer Spectrum 100 Fourier transform infrared spectrometer using $\mathrm{KBr}$ pellets.

\subsection{Electrochemical Measurements}

The electrochemical experiments were performed using a standard three-electrode configuration with the as-synthesized sample electrode as the working electrode, platinum as the counter electrode, and $\mathrm{Hg} / \mathrm{HgO}$ as the reference electrode. The electrolyte was a $2 \mathrm{~mol} \mathrm{~L}^{-1}$ aqueous $\mathrm{KOH}$ solution. The working electrodes were prepared as follows: $75 \mathrm{wt} \%$ active materials were mixed with $7.5 \mathrm{wt} \%$ acetylene black, $7.5 \mathrm{wt} \% \mathrm{KS}-6$, and $10 \mathrm{wt} \%$ polyvinylidene fluoride in NMP. The slurry was pressed on $\mathrm{Ni}$ foam $(2 \mathrm{~cm} \times 1 \mathrm{~cm} \times 1 \mathrm{~mm})$ and dried at $80{ }^{\circ} \mathrm{C}$ under vacuum for $6 \mathrm{~h}$. Each working electrode contained approximately $1 \mathrm{mg}$ of active material. $\mathrm{CV}$ and galvanostatic charge/ discharge tests were performed on an electrochemical workstation (Zahner Zennium CIMPS-1, Germany) in the potential range of $0-0.55 \mathrm{~V}$ and $0-0.45 \mathrm{~V}$, respectively. Electrochemical impedance spectroscopy (EIS) was carried out by applying a $5 \mathrm{mV}$ amplitude over a frequency range of $0.01 \mathrm{~Hz}$ to $100 \mathrm{kHz}$ at open circuit potential.

\section{Results and Discussion}

The crystal structure of the 3D Co-Al-LDHs calculated by the XRD pattern are shown in Fig. 1a. The diffraction peaks located at $11.3^{\circ}, 22.8^{\circ}, 35.0^{\circ}, 39.3^{\circ}, 46.4^{\circ}, 61.1^{\circ}$, and $62.4^{\circ}$ correspond to the (003), (006), (012), (015), (018), (110), and (113) facets, respectively, implying the obtained LDH product has a rhombohedral structure [39]. The XRD patterns (Fig. S1a, b) of the obtained 2D Co-Al-LDHs and OD Co-Al-LDHs show similar structures. SEM images (Figs. 1b, S2a, b) clearly show the 3D hierarchical structure built up of nanosheets. TEM and HRTEM images (Fig. 1d) further reveal the ultrathin nature with a thickness of approximately $1.6 \mathrm{~nm}$, which can also be confirmed by AFM measurements (Fig. S2c, d). 2D nanosheets with a thickness of approximately $2.5 \mathrm{~nm}$ (2D Co-Al-LDHs) and nanospheres with an overall size of approximately $50 \mathrm{~nm}$
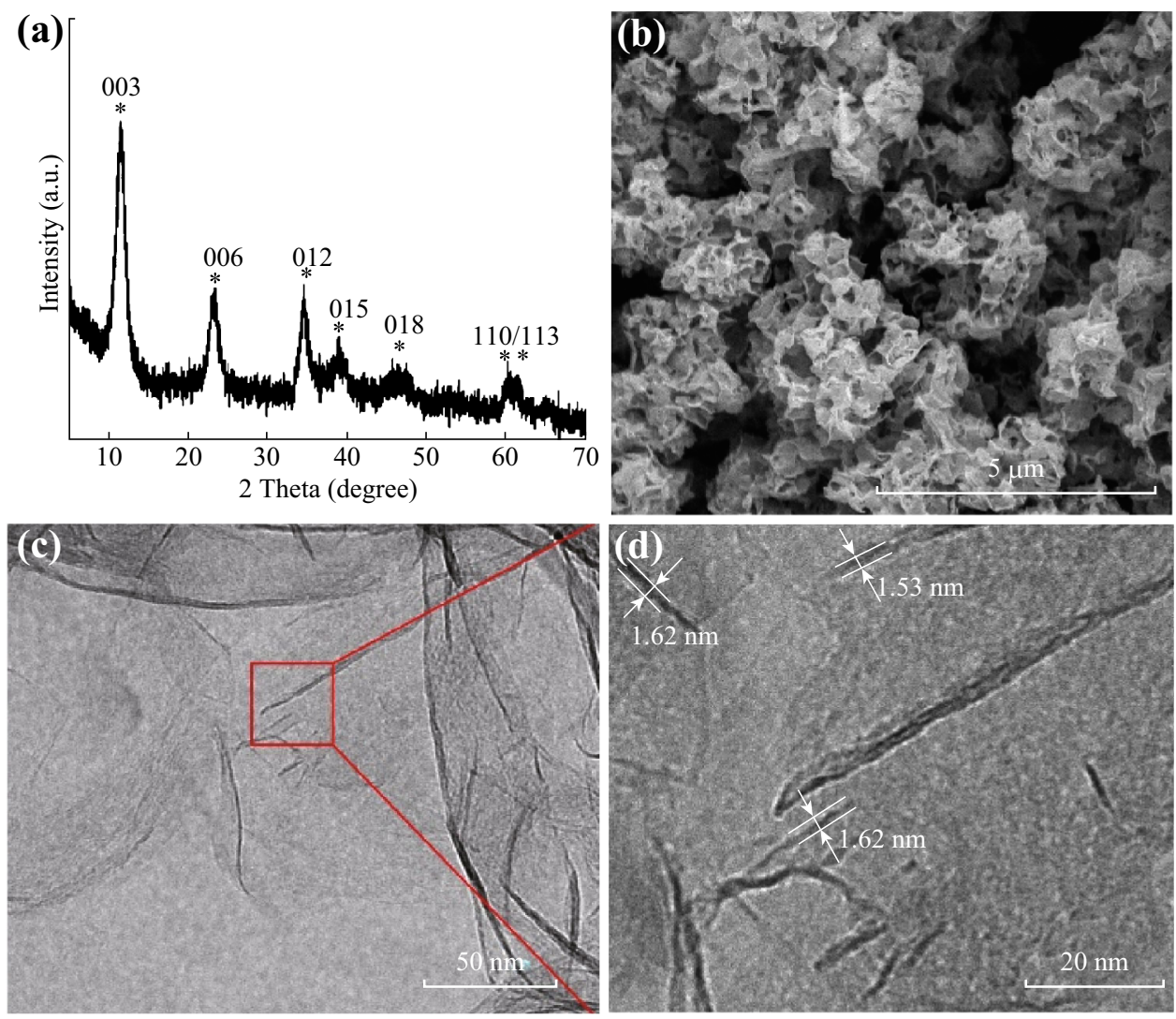

Fig. 1 a XRD pattern, b SEM image, c TEM image, and d HRTEM image of the as-prepared 3D Co-Al-LDHs 
(OD Co-Al-LDHs) were formed when the mixed solvent was replaced by deionized water or butyl alcohol, respectively (Fig. S1c-f). To evaluate the formation of 3D Co-AlLDHs, the XRD patterns and SEM images of the products prepared at different reaction times are shown in Fig. S3. The 2D Co-Al-LDH nanoplates were formed when the reaction time was $2 \mathrm{~h}$, and they gradually changed into selfassembled 3D Co-Al-LDHs built up of nanosheets with increasing reaction time. The morphological evolution from the $2 \mathrm{D} \mathrm{LDH}$ to the 3D LDH nanostructure follows co-precipitation, dissolution, and recrystallization processes. Furthermore, the selective adsorption of butyl alcohol on the $\{001\}$ facets of LDH can minimize the surface energy to form and stabilize the atomically thin LDH nanosheets [33-36]. The interactions of butyl alcohol adsorbed on the surface of the LDH nanosheets can also help form 3D microspheres by the self-assembly of atomically thin (mono-/bi-layers) LDH nanosheets.

$\mathrm{N}_{2}$ adsorption-desorption isotherms of 3D Co-AlLDHs, 2D Co-Al-LDHs, and 0D Co-Al-LDHs are shown in Fig. 2a. The mesoporous size of 3D Co-Al-LDHs is in the range of $3-10 \mathrm{~nm}$ (inset in Fig. 2a). Moreover, the specific surface area of the 3D Co-Al-LDH hierarchical structure is $152 \mathrm{~m}^{2} \mathrm{~g}^{-1}$, and the total pore volume is $0.52 \mathrm{~cm}^{3} \mathrm{~g}^{-1}$, which are much higher than those of the other samples (Table S2) and previously reported results [40-42]. Figure 2b depicts the FT-IR spectra of all samples. The broad adsorption peak at $3465 \mathrm{~cm}^{-1}$ is attributed to $\mathrm{O}-\mathrm{H}$ stretching modes of interlayer water molecules and $\mathrm{H}$-bonded $\mathrm{OH}$ groups. The weak peak at $1640 \mathrm{~cm}^{-1}$ corresponds to the bending mode of water molecules. The strong peaks at 1358 and $766 \mathrm{~cm}^{-1}$ belong to the $v_{3}$ vibrational and bending modes of $\mathrm{CO}_{3}{ }^{2-}$, respectively [43]. The weak absorption peaks in the range of $800-500 \mathrm{~cm}^{-1}$ correspond to the lattice vibrations of the $\mathrm{M}-\mathrm{O}$ and $\mathrm{O}-\mathrm{M}-$ $\mathrm{O}$ (where $\mathrm{M}=\mathrm{Co}, \mathrm{Al}$ ) groups [44]. The faint peaks at 2980 and $1055 \mathrm{~cm}^{-1}$ (Fig. S4) in the spectra of 3D Co-Al-
LDHs and OD Co-Al-LDHs belong to $\mathrm{C}-\mathrm{H}$ and $\mathrm{C}-\mathrm{C}$ or alkoxy groups (blue dashed line in Fig. 2b), indicating the presence of organic molecules (butyl alcohol) in the samples prepared in mixed solvent or butyl alcohol. No such peaks are detected in the spectrum of $2 \mathrm{D}$ Co-Al-LDHs prepared in water, further supporting the existence of butyl alcohol in 3D Co-Al-LDHs and OD Co-Al-LDHs.

The XPS spectrum of 3D Co-Al-LDHs shown in Fig. 3a indicates the presence of $\mathrm{Co}, \mathrm{Al}, \mathrm{O}$, and $\mathrm{C}$. The high-resolution XPS spectrum of Co (Fig. 3b) displays the spinorbit splitting of Co $2 p$ into Co $2 p^{1 / 2}(797.2$ and $803.1 \mathrm{eV})$ and $\mathrm{Co} 2 p^{3 / 2}$ (780.9 and $\left.786.6 \mathrm{eV}\right)$, suggesting the coexistence of $\mathrm{Co}^{2+}$ and $\mathrm{Co}^{3+}$ [45]. Additionally, the $\mathrm{C} 1 \mathrm{~s}$ peak can be separated into to five peaks centered at 284.6, $285.3,286.4,288.2$, and $289.4 \mathrm{eV}$, which may be attributed to $s p^{2} \mathrm{C}, s p^{3} \mathrm{C}, \mathrm{C}-\mathrm{O}, \mathrm{C}=\mathrm{O}$, and $\mathrm{O}=\mathrm{C}-\mathrm{O}$, respectively, derived from the adsorbed organic molecules and $\mathrm{CO}_{3}{ }^{2-}$ groups (see Fig. 3c). The peak at $74.2 \mathrm{eV}$ in the fine spectrum of $\mathrm{Al} 2 p$ is related to the $\mathrm{Al}^{3+}$ species in the form of $\mathrm{Al}-\mathrm{OH}$ [46].

The electrochemical energy storage performances of the obtained samples were studied by a three-electrode cell in the potential range of $0-0.55 \mathrm{~V}$ with $2 \mathrm{M} \mathrm{KOH}$ aqueous solution as the electrolyte. The specific capacitance of an electrode can be calculated using the following Eq. 1:

$C_{\mathrm{SP}}=I \times t /(\Delta V \times m)$,

where $I, t, \Delta V$, and $m$ stand for the constant current density $\left(\mathrm{A} \mathrm{g}^{-1}\right)$, the discharge time (s), the potential (V), and the mass of the electroactive material, respectively. Cyclic voltammetry $(\mathrm{CV})$ curves at various scan rates are shown in Fig. 4a. The symmetrical oxidation-reduction peaks at different scan rates imply the high electrochemical reversibility of 3D Co-Al-LDHs. The specific capacitances of the 3D-Co-Al-LDHs are 838, 813, 801, 792, 783, 780, 753, 732, 715, and $677 \mathrm{~F} \mathrm{~g}^{-1}$ at 1, 2, 5, 10, 15, 20, 30, 50, 70, and $100 \mathrm{~A} \mathrm{~g}^{-1}$ (see Fig. 4b), which are higher than the
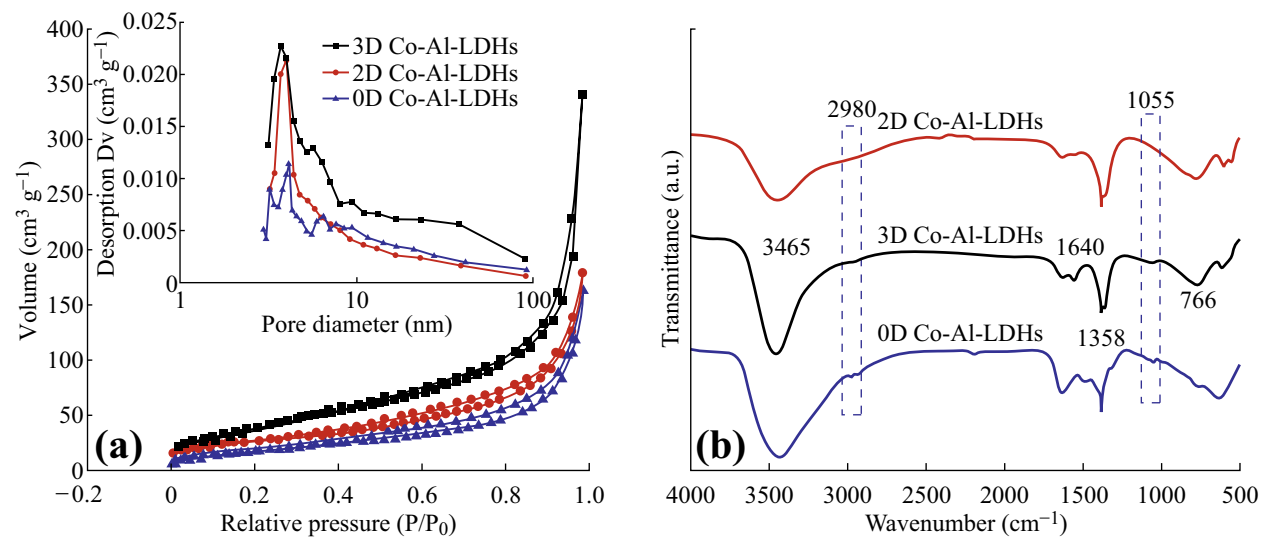

Fig. 2 a Nitrogen adsorption and desorption isotherms, and b FT-IR spectra of all Co-Al-LDHs samples, where the inset corresponds to the BJH pore size distribution of all Co-Al-LDH samples 

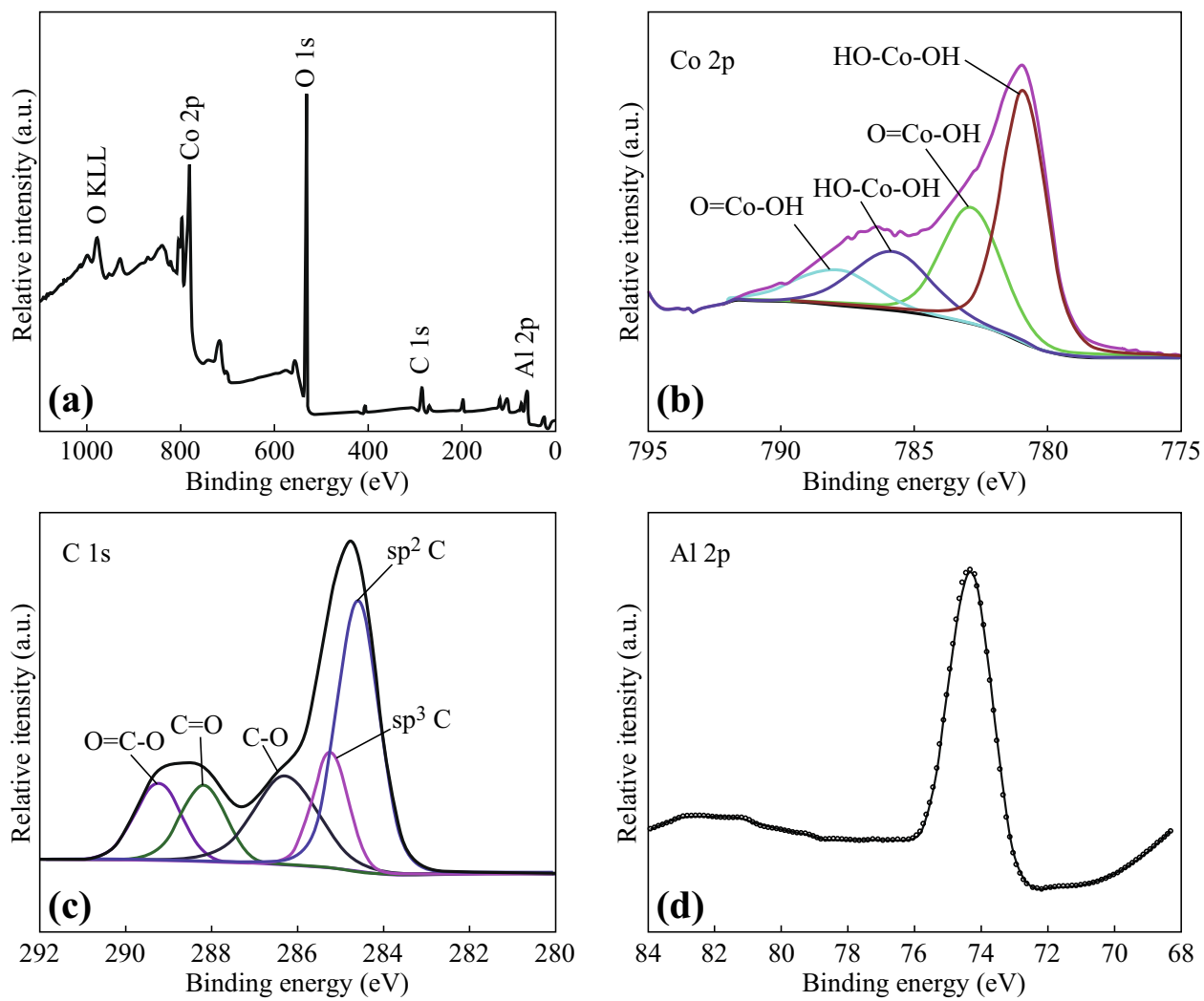

Fig. 3 a XPS spectrum of 3D Co-Al-LDHs. High-resolution spectra of b Co $2 p$, c C $1 s$, and d Al $2 p$

values of the most often reported carbon-based Co-AlLDH composites [23-25, 41, 47-49] (Fig. 4c; Table S1). The improved reversibility and rate capability of the $3 \mathrm{D}$ Co-Al-LDHs are derived from its abundant active sites and pores in the 3D hierarchical structure, which can further provide accessible pathways for electrolyte and facilitate the transport of ions from the liquid to the LDH. As shown in Fig. 4d, the 3D Co-Al-LDH electrode has a constant capacitance of $801 \mathrm{~F} \mathrm{~g}^{-1}$ in the initial 100 cycles at $5 \mathrm{~A} \mathrm{~g}^{-1}$, and maintains stable retentions of 99,98 , and $97 \%$ after every 100 cycles at 10,15 , and $20 \mathrm{~A} \mathrm{~g}^{-1}$, respectively. Furthermore, the 3D Co-Al-LDH electrode also exhibits long-term cycling stability and can still retain approximately $95 \%$ of the initial capacitance even after 20,000 cycles (Fig. 4e). The similar potential response of each charge-discharge curve also indicates the high reversibility of the charge-discharge process (inset in Fig. 4e).

To further understand the effects of the unique structure on the electrochemical performance of 3D Co-Al-LDHs, the $\mathrm{CV}$ and galvanostatic charge-discharge curves of $2 \mathrm{D}$ Co-Al-LDHs and OD Co-Al-LDHs were also determined, and the results are shown in Figs. S5 and S6. From Fig. S6, one can see that the 0D Co-Al-LDH nanoparticles had an initial capacitance of only $\sim 250 \mathrm{~F} \mathrm{~g}^{-1}$, while $3 \mathrm{D} \mathrm{Co}-\mathrm{Al}-$
LDHs and 2D Co-Al-LDHs had initial capacitances of $\sim 800 \mathrm{~F} \mathrm{~g}^{-1}$. The same initial capacitance of 3D and 2D Co-Al-LDHs is due to the similar 2D atomically thin structure. As shown in Fig. 5a, the capacitance of 2D CoAl-LDHs decreases rapidly to $450 \mathrm{~F} \mathrm{~g}^{-1}$ (only $44 \%$ of the original value) after 5000 cycles at $5 \mathrm{~A} \mathrm{~g}^{-1}$ due to the restacking of 2D nanosheets, while 3D Co-Al-LDHs retains 93\% of the initial specific capacitance (from 801 to $745 \mathrm{~F} \mathrm{~g}^{-1}$ ) and 0D Co-Al-LDHs retains almost $100 \%$. The difference is related to the dispersion solution; that is, 2D Co-Al-LDHs were prepared in water, whereas 3D Co$\mathrm{Al}-\mathrm{LDH}$ and $0 \mathrm{D} \mathrm{Co}-\mathrm{Al}-\mathrm{LDHs}$ were prepared in mixed solvent or butyl alcohol. The surface of the $3 \mathrm{D}$ and $0 \mathrm{D} \mathrm{Co}-$ Al-LDHs are modified by organic molecules, as supported by the FT-IR (Fig. 2b) and XPS (Fig. 3) spectra. The organic molecules adsorbed at the surface reduce the surface energy and improve the stability of the electrode materials [32-36]. EIS spectra of the electrodes were taken before and after the cycling process (Fig. $5 \mathrm{~b}, \mathrm{c}$ ). High charge transfer and diffusion resistance were observed in 0D Co-Al-LDHs, which therefore led to poor capacitance. Nearly the same diffusion resistance was observed in 3D Co-Al-LDHs, whereas it became larger after the cycling process in 2D Co-Al-LDHs. The changes in diffusion resistance indicate that the $3 \mathrm{D} \mathrm{LDH}$ structure can 
(a)
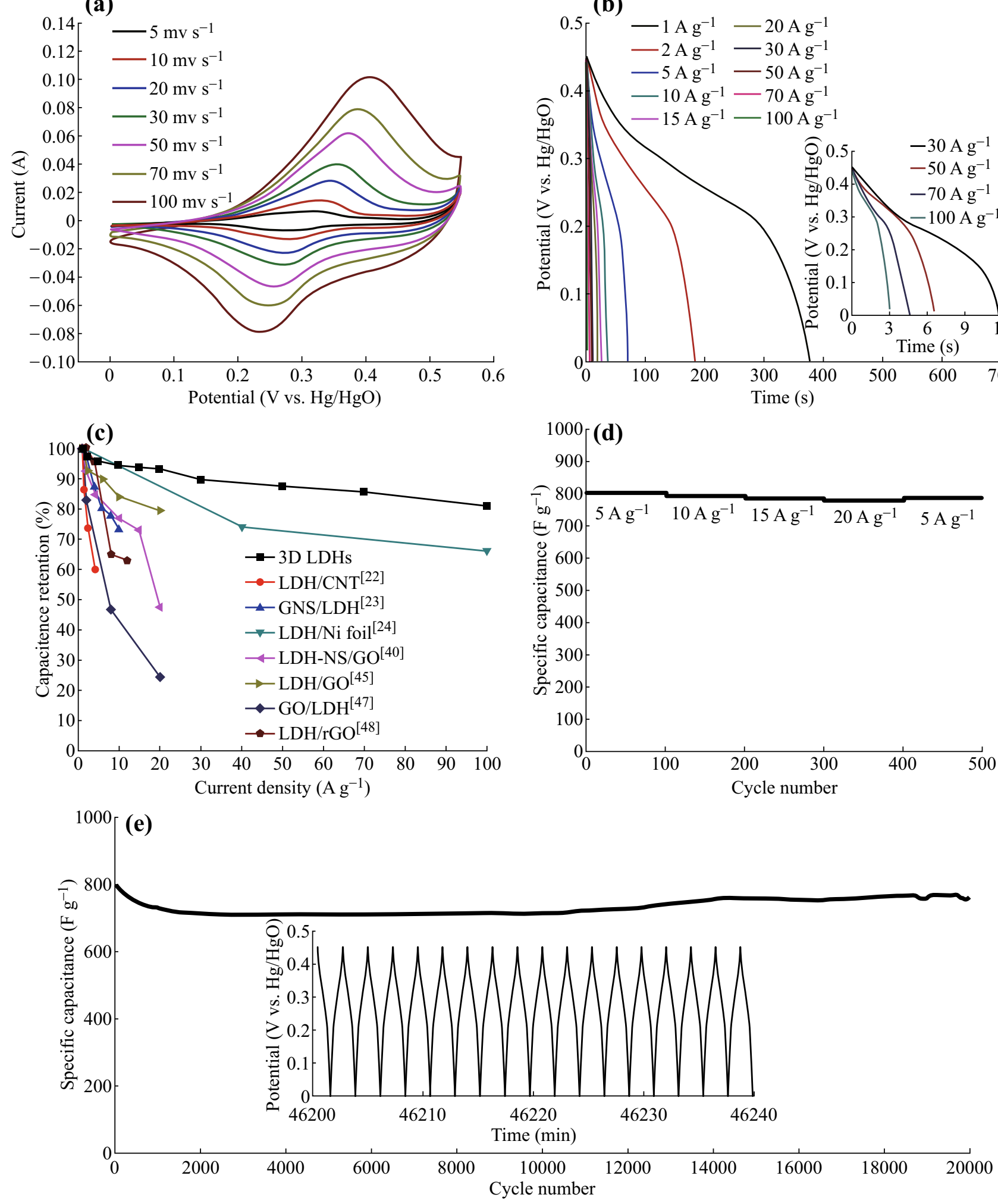

(b)
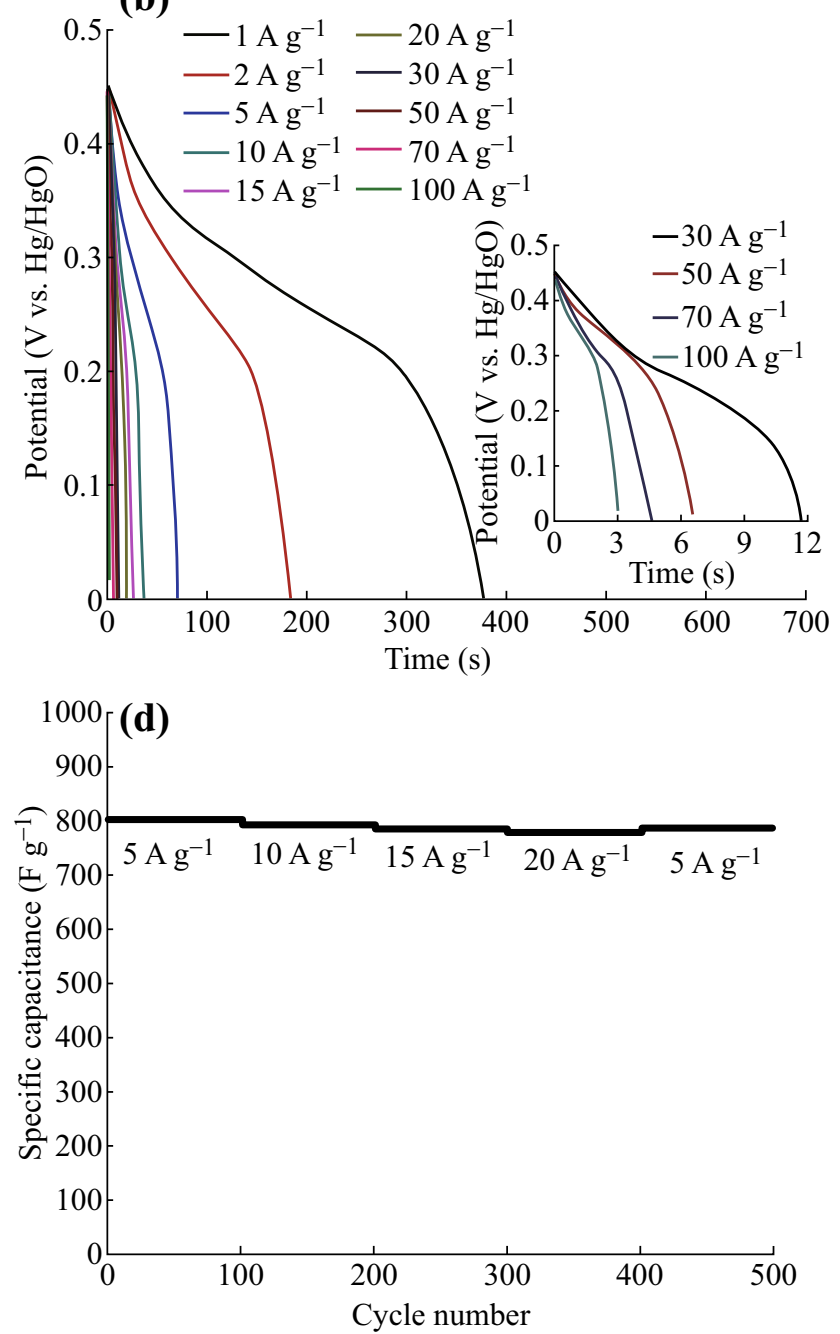

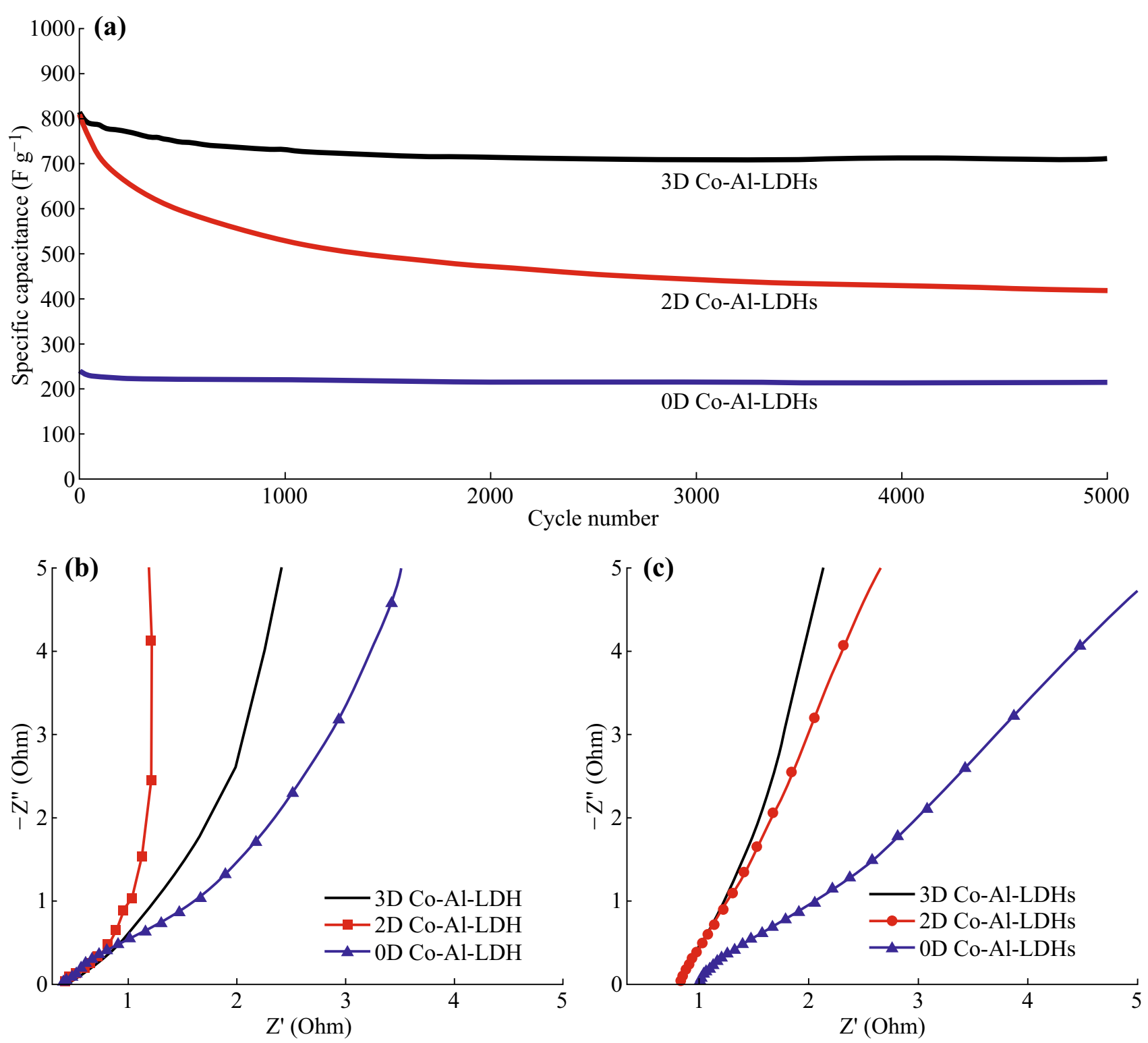

Fig. 5 a Cycling performances at $5 \mathrm{~A} \mathrm{~g}^{-1}$ and EIS spectrums before $\mathbf{b}$ and after $\mathbf{c} 1000$ cycles of all Co-Al-LDH samples

effectively prevent the re-stacking of 2D nanosheets, which further results in the unique cycling stability of the obtained 3D Co-Al-LDHs.

The high performance of the 3D Co-Al-LDH material can be ascribed to its unique 3D hierarchical structure. First, the atomically thin building units with thicknesses of approximately $1.6 \mathrm{~nm}\left(152 \mathrm{~m}^{2} \mathrm{~g}^{-1}\right)$ can provide a large amount of electrochemically active sites to result in high capacitance. Additionally, the 3D hierarchical structures can prevent the re-stacking of nanosheets, and the surface modification of organic molecules can enhance the stability of 3D Co-Al-LDHs (Figs. S7, S8), leading to long-term cyclic stability. Furthermore, the pores in the 3D hierarchical structure are readily accessible for electrolyte, facilitating the transport of ions from the liquid to the active surface of the LDH. Finally, the 3D hierarchical structure can also supply more points to connect the conductive matrix in the electrode, which is beneficial to the conductivity and rate capability of the electrodes.

\section{Conclusion}

A facile synthetic route was developed to directly prepare 3D hierarchical Co-Al-LDHs composed of atomically thin nanosheets. The as-obtained hierarchical Co-Al-LDHs show a high specific capacitance of $801 \mathrm{~F} \mathrm{~g}^{-1}$ at $5 \mathrm{~A} \mathrm{~g}^{-1}$, excellent rate performance with a capacitance of $677 \mathrm{~F} \mathrm{~g}^{-1}$ 
at $100 \mathrm{~A} \mathrm{~g}^{-1}$, and good cycling stability with only $5 \%$ decline after 20,000 cycles. Such excellent performance is derived from its atomically thin building units modified by organic molecules and its unique 3D hierarchical structure. This work may provide a promising alternative strategy to prepare other LDHs with enhanced electrochemical properties for supercapacitors.

Acknowledgements This work was supported by the National Basic Research Program of China (2014CB239702), Research project of environmental protection in Jiangsu province (2016060), and Science and Technology Commission of Shanghai Municipality (14DZ2250800).

Open Access This article is distributed under the terms of the Creative Commons Attribution 4.0 International License (http://crea tivecommons.org/licenses/by/4.0/), which permits unrestricted use, distribution, and reproduction in any medium, provided you give appropriate credit to the original author(s) and the source, provide a link to the Creative Commons license, and indicate if changes were made.

\section{References}

1. S. Tsujimura, E. Suraniti, F. Durand, N. Mano, Oxygen reduction reactions of the thermostable bilirubin oxidase from Bacillus pumilus on mesoporous carbon-cryogel electrodes. Electrochim. Acta 117, 263-267 (2014). doi:10.1016/j.electacta.2013.11.131

2. C.H. Tang, X.S. Yin, H. Gong, A study on dramatically enhanced capacitance of graphene-decorated hierarchically porous nickelian heterogenite for energy storage application. Electrochim. Acta 114, 543-550 (2013). doi:10.1016/j.electacta.2013.10.065

3. H.Y. Sun, Y.G. Liu, Y.L. Yu, M. Ahmad, D. Nan, J. Zhu, Mesoporous $\mathrm{Co}_{3} \mathrm{O}_{4}$ nanosheets-3D graphene networks hybrid materials for high-performance lithium ion batteries. Electrochim. Acta 118, 1-9 (2014). doi:10.1016/j.electacta.2013.11.181

4. Q. Wang, J. Yan, Y. Xiao, T. Wei, Z.J. Fan, M.L. Zhang, X.Y. Jing, Interconnected porous and nitrogen-doped carbon network for supercapacitors with high rate capability and energy density. Electrochim. Acta 114, 165-172 (2013). doi:10.1016/j.electacta. 2013.10.044

5. X.J. Bo, M. Li, C. Han, L.P. Guo, The influence of boron dopant on the electrochemical properties of graphene as an electrode material and a support for Pt catalysts. Electrochim. Acta 114, 582-589 (2013). doi:10.1016/j.electacta.2013.10.088

6. P. Simon, Y. Gogotsi, Materials for electrochemical capacitors. Nat. Mater. 7(11), 845-854 (2008). doi:10.1038/nmat2297

7. Y. Zhao, J. Liu, Y. Hu, H.H. Cheng, C.G. Hu, C.C. Jiang, L. Jiang, A.Y. Cao, L.T. Qu, Highly compression-tolerant supercapacitor based on polypyrrole-mediated graphene foam electrodes. Adv. Mater. 25(4), 591-595 (2013). doi:10.1002/adma. 201203578

8. Z.J. Fan, J. Yan, T. Wei, L.J. Zhi, G.Q. Ning, T.Y. Li, F. Wei, Asymmetric supercapacitors based on graphene/ $\mathrm{MnO}_{2}$ and activated carbon nanofiber electrodes with high power and energy density. Adv. Funct. Mater. 21(12), 2366-2375 (2011). doi:10. 1002/adfm.201100058

9. X.L. Li, Q. Song, L. Hao, L.J. Zhi, Graphenal polymers for energy storage. Small 10(11), 2122-2135 (2014). doi:10.1002/ smll.201303717
10. X. Yang, K. Xu, R. Zou, J. Hu, A hybrid electrode of $\mathrm{Co}_{3-}$ $\mathrm{O}_{4} @ \mathrm{PPy}$ core/shell nanosheet arrays for high-performance supercapacitors. Nano-Micro Lett. 8(2), 143-150 (2016). doi:10. 1007/s40820-015-0069-x

11. H.L. Wang, H.S. Casalongue, Y.Y. Liang, H.J. Dai, Ni(OH $)_{2}$ nanoplates grown on graphene as advanced electrochemical pseudocapacitor materials. J. Am. Chem. Soc. 132(21), 7472-7477 (2010). doi:10.1021/ja102267j

12. R. Kumar, R.K. Singh, P.K. Dubey, D.P. Singh, R.M. Yadav, R.S. Tiwari, Hydrothermal synthesis of a uniformly dispersed hybrid graphene- $\mathrm{TiO}_{2}$ nanostructure for optical and enhanced electrochemical applications. RSC Adv. 5(10), 7112-7120 (2015). doi:10.1039/C4RA06852A

13. R. Kumar, R.K. Singh, R. Savu, P.K. Dubey, P. Kumar, S.A. Moshkalev, Microwave-assisted synthesis of void-induced graphene-wrapped nickel oxide hybrids for supercapacitor applications. RSC Adv. 6(32), 26612-26620 (2016). doi:10.1039/ C6RA00426A

14. R. Kumar, R.K. Singh, P.K. Dubey, D.P. Singh, R.M. Yadav, Self-assembled hierarchical formation of conjugated 3D cobalt oxide nanobead-CNT-graphene nanostructure using microwaves for high-performance supercapacitor electrode. ACS Appl. Mater. Interfaces 7(27), 15042-15051 (2015). doi:10.1021/acsami. $5 b 04336$

15. K. Nagaoka, K. Takanabe, K. Aika, Modification of $\mathrm{Co} / \mathrm{TiO}_{2}$ for dry reforming of methane at $2 \mathrm{MPa}$ by Pt, Ru or Ni. Appl. Catal. A 268(1-2), 151-158 (2004). doi:10.1016/j.apcata.2004.03.029

16. Z.Y. Sun, C.G. Lin, J.Y. Zheng, L. Wang, J.W. Zhang, F.L. Xu, J. Hou, Fabrication and characterization of hierarchical $\mathrm{Mg} / \mathrm{Ni} / \mathrm{Al}$ layered double hydroxide framework on aluminum foam. Mater. Lett. 113, 83-86 (2013). doi:10.1016/j.matlet.2013.09.063

17. Y.P. Wan, W.R. Zhao, Y. Tang, L. Li, H.J. Wang, Y.L. Cui, J.L. Gu, Y.S. Li, J.L. Shi, Ni-Mn bi-metal oxide catalysts for the low temperature SCR removal of $\mathrm{NO}$ with $\mathrm{NH}_{3}$. Appl. Catal. B 148, 114-122 (2014). doi:10.1016/j.apcatb.2013.10.049

18. X.M. Li, J.T. Zai, Y.Y. Liu, X.B. He, S.J. Xiang, Z.F. Ma, X.F. Qian, Atomically thin layered NiFe double hydroxides assembled 3D microspheres with promoted electrochemical performances. J. Power Sources 325, 675-681 (2016). doi:10.1016/j.jpowsour. 2016.06.090

19. V. Gupta, S. Gupta, N. Miura, Al-substituted alpha-cobalt hydroxide synthesized by potentiostatic deposition method as an electrode material for redox-supercapacitors. J. Power Sources 177(2), 685-689 (2008). doi:10.1016/j.jpowsour.2007.10.091

20. Y. Wang, W.S. Yang, C. Chen, D.G. Evans, Fabrication and electrochemical characterization of cobalt-based layered double hydroxide nanosheet thin-film electrodes. J. Power Sources 184(2), 682-690 (2008). doi:10.1016/j.jpowsour.2008.02.017

21. P.C.K. Vesborg, T.F. Jaramillo, Addressing the terawatt challenge: scalability in the supply of chemical elements for renewable energy. RSC Adv. 2(21), 7933-7947 (2012). doi:10.1039/ c2ra20839c

22. L. Yu, N.N. Shi, Q. Liu, J. Wang, B. Yang, B. Wang, H.J. Yan, Y.B. Sun, X.Y. Jing, Facile synthesis of exfoliated Co-Al LDHcarbon nanotube composites with high performance as supercapacitor electrodes. Phys. Chem. Chem. Phys. 16(33), 17936-17942 (2014). doi:10.1039/C4CP02020K

23. L.J. Zhang, X.G. Zhang, L.F. Shen, B. Gao, L. Hao, X.J. Lu, F. Zhang, B. Ding, C.Z. Yuan, Enhanced high-current capacitive behavior of graphene/CoAl-layered double hydroxide composites as electrode material for supercapacitors. J. Power Sources 199, 395-401 (2012). doi:10.1016/j.jpowsour.2011.10.056

24. X.L. Wu, L.L. Jiang, C.L. Long, T. Wei, Z.J. Fan, Dual support system ensuring porous Co-Al hydroxide nanosheets with ultrahigh rate performance and high energy density for 
supercapacitors. Adv. Funct. Mater. 25(11), 1648-1655 (2015). doi:10.1002/adfm.201404142

25. X.X. Liu, A. Zhou, T. Pan, Y.B. Dou, M.F. Shao, J.B. Han, M. Wei, Ultrahigh-rate-capability of a layered double hydroxide supercapacitor based on a self-generated electrolyte reservoir. J. Mater. Chem. A 4(21), 8421-8427 (2016). doi:10.1039/ C6TA02164F

26. J.W. Zhao, Z.Z. Lu, M.F. Shao, D.P. Yan, M. Wei, D.G. Evans, $\mathrm{X}$. Duan, Flexible hierarchical nanocomposites based on $\mathrm{MnO}_{2}$ nanowires/CoAl hydrotalcite/carbon fibers for high-performance supercapacitors. RSC Adv. 3(4), 1045-1049 (2013). doi:10.1039/ C2RA22566B

27. H. Chen, L.F. Hu, M. Chen, Y. Yan, L.M. Wu, Nickel- cobalt layered double hydroxide nanosheets for high- performance supercapacitor electrode materials. Adv. Funct. Mater. 24(7), 934-942 (2014). doi:10.1002/adfm.201301747

28. J. Zai, K. Wang, Y. Su, X. Qian, J. Chen, High stability and superior rate capability of three-dimensional hierarchical $\mathrm{SnS}_{2}$ microspheres as anode material in lithium ion batteries. J. Power Sources 196(7), 3650-3654 (2011). doi:10.1016/j.jpowsour.2010. 12.057

29. J. Hu, M. Chen, X.S. Fang, L.M. Wu, Fabrication and application of inorganic hollow spheres. Chem. Soc. Rev. 40(11), 5472-5491 (2011). doi:10.1039/c1cs15103g

30. Y. Zhao, L.F. Hu, S.Y. Zhao, L.M. Wu, Preparation of $\mathrm{MnCo}_{2-}$ $\mathrm{O}_{4} @ \mathrm{Ni}(\mathrm{OH})_{2}$ core-shell flowers for asymmetric supercapacitor materials with ultrahigh specific capacitance. Adv. Funct. Mater. 26(23), 4085-4093 (2016). doi:10.1002/adfm.201600494

31. S. Huang, Q. He, W. Chen, J. Zai, Q. Qiao, X. Qian, 3D hierarchical $\mathrm{FeSe}_{2}$ microspheres: controlled synthesis and applications in dye-sensitized solar cells. Nano Energy 15, 205-215 (2015). doi:10.1016/j.nanoen.2015.04.027

32. W. Chen, M. Wang, T. Qian, H. Cao, S. Huang, Q. He, N. Liang, C. Wang, J. Zai, Rational design and fabrication of skeletal $\mathrm{Cu} 7 \mathrm{~S} 4$ nanocages for efficient counter electrode in quantum dotsensitized solar cells. Nano Energy 12, 186-196 (2015). doi:10. 1016/j.nanoen.2014.12.026

33. L. Carbone, P.D. Cozzoli, Colloidal heterostructured nanocrystals: synthesis and growth mechanisms. Nano Today 5(5), 449-493 (2010). doi:10.1016/j.nantod.2010.08.006

34. N.P. Herring, K. AbouZeid, M.B. Mohamed, J. Pinsk, M.S. ElShall, Formation mechanisms of gold-zinc oxide hexagonal nanopyramids by heterogeneous nucleation using microwave synthesis. Langmuir 27(24), 15146-15154 (2011). doi:10.1021/ la201698k

35. Z. Niu, Y. Li, Removal and utilization of capping agents in nanocatalysis. Chem. Mater. 26(1), 72-83 (2014). doi:10.1021/ $\mathrm{cm} 4022479$

36. B.L. Cushing, V.L. Kolesnichenko, C.J. O’Connor, Recent advances in the liquid-phase syntheses of inorganic nanoparticles. Chem. Rev. 104(9), 3893-3946 (2004). doi:10.1021/cr030027b

37. B. Nagendra, K. Mohan, E.B. Gowd, Polypropylene/layered double hydroxide (LDH) nanocomposites: influence of $\mathrm{LDH}$ particle size on the crystallization behavior of polypropylene. ACS Appl. Mater. Interfaces 7(23), 12399-12410 (2015). doi:10. 1021/am5075826

38. J. Xiao, X.J. Wang, X.Q. Yang, S.D. Xun, G. Liu, P.K. Koech, J. Liu, J.P. Lemmon, Electrochemically induced high capacity displacement reaction of $\mathrm{PEO} / \mathrm{MoS}_{2} /$ graphene nanocomposites with lithium. Adv. Funct. Mater. 21(15), 2840-2846 (2011). doi:10.1002/adfm.201002752

39. Z.Y. Lu, W. Zhu, X.D. Lei, G.R. Williams, D. O'Hare, Z. Chang, X.M. Sun, X. Duan, High pseudocapacitive cobalt carbonate hydroxide films derived from CoAl layered double hydroxides. Nanoscale 4(12), 3640-3643 (2012). doi:10.1039/c2nr30617d

40. C.C. Wang, H.C. Chen, S.Y. Lu, Manganese oxide/graphene aerogel composites as an outstanding supercapacitor electrode material. Chem-Eur. J. 20(2), 517-523 (2014). doi:10.1002/chem. 201303483

41. A.L. Zhang, C. Wang, Q.J. Xu, H.M. Liu, Y.G. Wang, Y.Y. Xia, A hybrid aerogel of Co-Al layered double hydroxide/graphene with three-dimensional porous structure as a novel electrode material for supercapacitors. RSC Adv. 5(33), 26017-26026 (2015). doi:10.1039/C5RA00103J

42. J.W. Zhao, J. Chen, S.M. Xu, M.F. Shao, Q. Zhang et al., Hierarchical NiMn layered double hydroxide/carbon nanotubes architecture with superb energy density for flexible supercapacitors. Adv. Funct. Mater. 24(20), 2938-2946 (2014). doi:10.1002/ adfm. 201303638

43. Z.P. Liu, R.Z. Ma, M. Osada, N. Iyi, Y. Ebina, K. Takada, T. Sasaki, Synthesis, anion exchange, and delamination of Co-Al layered double hydroxide: assembly of the exfoliated nanosheet/ polyanion composite films and magneto-optical studies. J. Am. Chem. Soc. 128(14), 4872-4880 (2006). doi:10.1021/ja0584471

44. H.D. Peng, Y. Han, T.X. Liu, W.C. Tjiu, C.B. He, Morphology and thermal degradation behavior of highly exfoliated Co-Allayered double hydroxide/polycaprolactone nanocomposites prepared by simple solution intercalation. Thermochim. Acta 502(1-2), 1-7 (2010). doi:10.1016/j.tca.2010.01.009

45. S. Thimmaiah, M. Rajamathi, N. Singh, P. Bera, F. Meldrum, N. Chandrasekhar, R. Seshadri, A solvothermal route to capped nanoparticles of gamma- $\mathrm{Fe}_{2} \mathrm{O}_{3}$ and $\mathrm{CoFe}_{2} \mathrm{O}_{4}$. J. Mater. Chem. 11(12), 3215-3221 (2001). doi:10.1039/b104070g

46. Y.F. Gao, A. Nagai, Y. Masuda, F. Sato, W.S. Seo, K. Koumoto, Surface precipitation of highly porous hydrotalcite-like film on Al from a zinc aqueous solution. Langmuir 22(8), 3521-3527 (2006). doi:10.1021/la052424i

47. J.H. Fang, M. Li, Q.Q. Li, W.F. Zhang, Q.L. Shou, F. Liu, X.B. Zhang, J.P. Cheng, Microwave-assisted synthesis of Co-Al-layered double hydroxide/graphene oxide composite and its application in supercapacitors. Electrochim. Acta 85, 248-255 (2012). doi:10.1016/j.electacta.2012.08.078

48. L. Wang, D. Wang, X.Y. Dong, Z.J. Zhang, X.F. Pei, X.J. Chen, B.A. Chen, J.A. Jin, Layered assembly of graphene oxide and CoAl layered double hydroxide nanosheets as electrode materials for supercapacitors. Chem. Commun. 47(12), 3556-3558 (2011). doi:10.1039/c0cc05420h

49. J.H. Hao, W.S. Yang, Z. Zhang, B.P. Lu, X. Ke, B.L. Zhang, J.L. Tang, Facile synthesis of three dimensional hierarchical Co-Al layered double hydroxides on graphene as high-performance materials for supercapacitor electrode. J. Colloid Interface Sci. 426, 131-136 (2014). doi:10.1016/j.jcis.2014.04.003 